
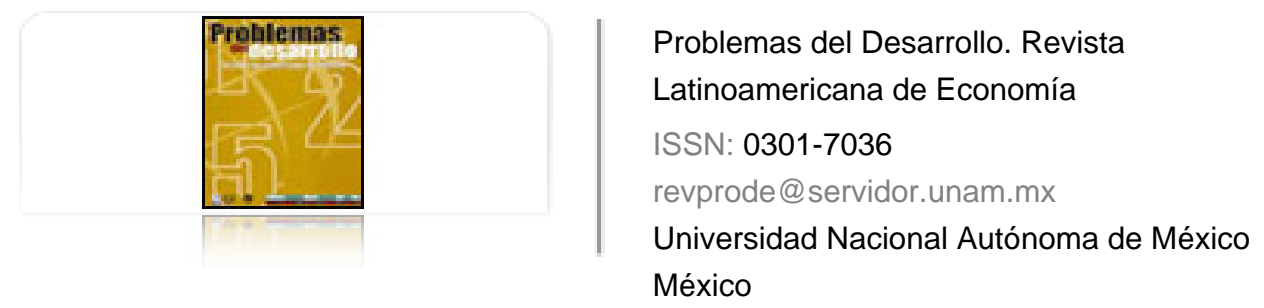

Pengue, Walter A.

CUESTIONES ECONÓMICO-AMBIENTALES DE LAS TRANSFORMACIONES AGRÍCOLAS EN LAS

PAMPAS

Problemas del Desarrollo. Revista Latinoamericana de Economía, vol. 40, núm. 157, abril-junio, 2009, pp. 137-161

Universidad Nacional Autónoma de México

Distrito Federal, México

Disponible en: http://www.redalyc.org/articulo.oa?id=11820087002

Cómo citar el artículo

Número completo

- Más información del artículo

Página de la revista en redalyc.org

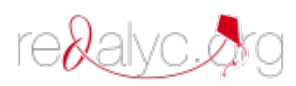

Sistema de Información Científica

Red de Revistas Científicas de América Latina, el Caribe, España y Portugal Proyecto académico sin fines de lucro, desarrollado bajo la iniciativa de acceso abierto 


\title{
CUESTIONES ECONÓMICO-AMBIENTALES DE LAS TRANSFORMACIONES AGRICOLAS EN LAS PAMPAS
}

\author{
Walter A. Pengue*
}

Fecha de recepción: 9 de febrero de 2009. Fecha de aceptación: 9 de mayo de 2009.

\section{Resumen}

En las últimas dos décadas, la agricultura argentina ha tenido transformaciones que cambiaron definitivamente su perfil agroproductivo. La incorporación de la siembra directa como práctica conservacionista, junto con su paquete asociado del herbicida glifosato, y el cultivo de soja transgénica son los pilares en los que se concentró este modelo, intensificado tanto hacia la agricultura (agriculturización) como hacia la ganadería intensiva (feedlot), todo ello sin considerar prácticamente las externalidades producidas. Estos problemas ambientales se reflejan en una importante pérdida de nutrientes y degradación de suelos, la destrucción de hábitats, el aumento en las exportaciones de agua virtual, la aparición de nuevas plagas, enfermedades y malezas tolerantes y resistentes como el sorgo de alepo, sumados a la deforestación en varias ecorregiones. Áreas con poblaciones importantes como en la cuenca del río Luján comienzan a recibir también estos impactos. El estudio se centra en un análisis sobre la evolución y situación de los nutrientes del suelo, como un indicador de pérdida de calidad ambiental, social y económica.

Palabras clave: extracción de nutrientes, agricultura intensiva, soja transgénica, glifosato, feedlots, deuda ambiental y río Luján.

* Director del Posgrado en Economía Ecológica, FADU, Universidad de Buenos Aires (UBA), y coordinador del grupo de Ecología del Paisaje y Medio Ambiente, GEPAMA, FADU, UBA. Correo electrónico: wapengue@ungs.edu.ar. 


\section{Abstract}

During the last two decades, Argentine agriculture has been transformed, definitively changing its agro-productive profile. The incorporation of direct sowing as a conservationist practice, together with its associated package of glyphosate herbicide and the cultivation of genetically-modified soy are the pillars on which this model is concentrated, intensifying both towards agriculture (agriculturalization) and stock-breeding (feedlot). The environmental problems are reflected in an important loss of nutrients, the rise in exports of virtual water, the appearance of new plagues, illnesses and resistant weeds and the deforestation of several eco-regions. Even areas of high population like the River Luján Basin are beginning to receive these impacts. The study is focused on an analysis of the evolution and situation of soil nutrients, as an indicator of the loss of environmental social and economic quality.

Key words: extraction of nutrients, Intensive agriculture, genetically-modified soy, glyphosate, feedlots, ecological debt, River Luján

\section{Résumé}

Au tours de dernières décennies, l'agriculture argentine a subi des transformations qui ont définitivement changé son profil agro-productif. L'incorporation des semailles directes comme pratique de conservation, jointes à la combinaison de l'herbicide glifosato et de la culture du soja transgénique, sont les piliers de ce modèle, qui intensifie autant l'agriculture (agriculturisation) que l'élevage (feedlot). Les problèmes environnementaux se reflètent dans un important appauvrissement des sols, l'augmentation virtuelle des exportations d'eau, l'apparition de nouveaux parasites et maladies ainsi que de mauvaises herbes résistantes, et la déforestation dans plusieurs éco-régions. Même des zones ayant des agglomérations importantes comme le bassin du fleuve Lujan commencent à subir ces impacts. L'étude se focalise sur une analyse de l'évolution et de l'état actuel des éléments nutritifs du sol, en tant qu'ils témoignent de la dégradation environnementale, sociale et économique.

Mots clés: extraction d'éléments nutritifs, agriculture intensive, soja transgénique, glifosato, feedlots, dette écologique, fleuve Lujan

\section{Resumo}

Nas últimas duas décadas, a agricultura argentina tem tido transformações, que mudaram definitivamente o seu perfil agroprodutivo. A incorporação do semeado no sistema de plantio direto como prática conservacionista, junto com o seu pacote associado do herbicida glifosato e o cultivo de soja transgênica, são os pilares nos que se concentrou este modelo, intensificado tanto em relação à agricultura (agriculturização) como à criação de gado (feedlot). Os problemas ambientais se refletem numa importante perda de nutrientes, o aumento nas exportações de água virtual, a aparição de novas pragas, enfermidades e de ervas daninhas resistentes e desmatamento em várias ecorregiões. Inclusive áreas com populações importantes como na Cuenca del Rio Luján começam a receber estes impactos. $O$ estudo se focaliza numa análise sobre a evolução e situação dos nutrientes do solo, como um indicador de perda de qualidade ambiental, social e econômica.

Palavras-chave: extração de nutrientes, agricultura intensiva, soja transgênica, glifosato, fedlots, dívida ecológica, rio Luján.

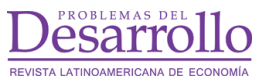


Introducción: cuestiones económicas de un modelo rural defectuoso

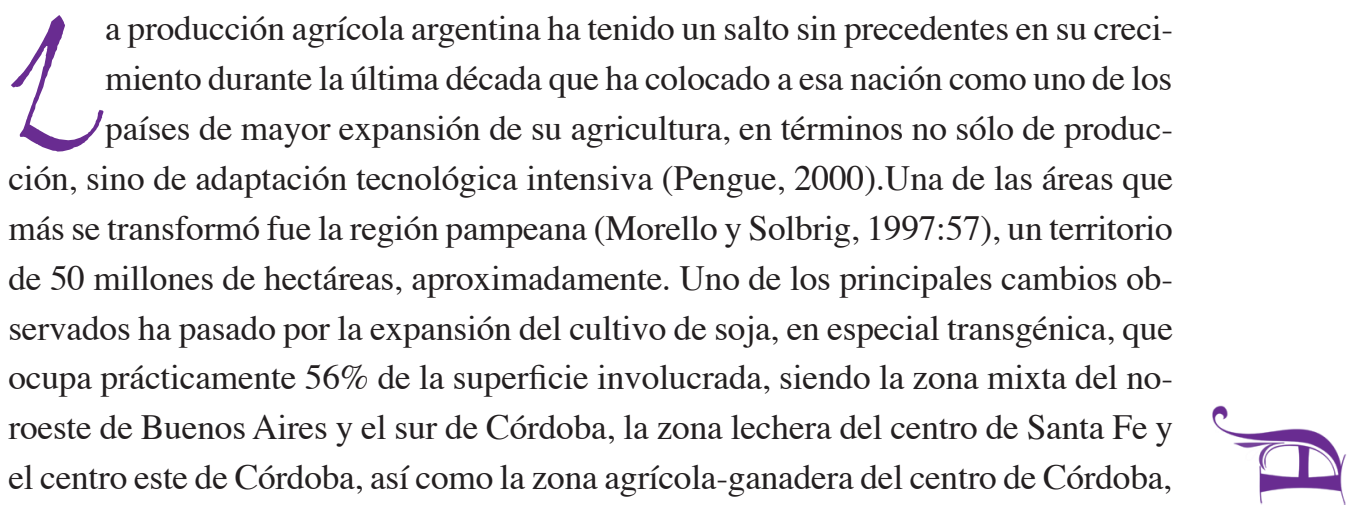
los espacios territoriales donde se ha producido la mayor expansión.

Otro cambio importante lo ha dado la llegada del modelo económico productivo de la siembra directa a principios de los años noventa, que por una parte permite desarrollar una práctica conservacionista al dejar rastrojo en superficie para la protección del suelo y, por otra, facilita un mejor flujo de caja, lo que posibilita al agricultor sembrar tres cultivos en dos años, en un modelo de rotación (trigo-soja de segundasoja) altamente rentable. La siembra directa se está expandiendo a un ritmo sostenido en todas las zonas de producción, sobre todo en el cultivo de soja. En la actualidad, según fuentes privadas (AAPRESID), algo más de 41\% del trigo, 50\% de maíz, 73\% de soja y $15 \%$ del girasol, se realizan en siembra directa.

Sin embargo, esta expansión de la soja alentada por los aspectos económicos pone en riesgo la sustentabilidad de las tierras agrícolas y amenaza a las áreas naturales. La soja es un cultivo con alta capacidad de extracción de nutrientes y la fertilización nitrogenada no es una práctica habitual; por otro lado, la cantidad y calidad de residuos que aporta es muy baja. Al ser una leguminosa, la soja fija nitrógeno, pero en condiciones intensivas como las del modelo sojero argentino éste no es suficiente. Como consecuencia, se producen balances negativos de carbono y nitrógeno que conducen a la disminución del contenido de materia orgánica de los suelos.

Del análisis de la influencia de variables económicas sobre el uso de la tierra y la elasticidad de sustitución entre cultivos en la región pampeana, se puede inferir que la decisión de sembrar los cultivos de trigo y maíz está estrechamente relacionada con el precio esperado del producto, en cambio para el cultivo de soja es el costo de implantación y producción el que más influye.

A medida que se reduce el costo de implantación se eleva proporcionalmente el área sembrada con soja. Si se tienen en cuenta las tendencias alcistas y bajistas de 
los costos de implantación y producción de maíz y soja respectivamente, es posible afirmar que parte de la expansión del cultivo de soja se debe a la reducción de sus costos de implantación. La tendencia al fuerte aumento de los precios internacionales de la commodity desde el año 2007 (que igual cayó a la mitad en los albores de 2009) ha sido un factor fuertemente traccionador para impulsar otro desplazamiento intenso hacia la soja, a pesar de la presión por las retenciones pensadas para ser aplicadas en la apropiación de la renta extraordinaria, no así para la captura de la renta ambiental. La retención es un impuesto aplicado por el gobierno a los agricultores, por medio de una quita (que realizan las compañías cerealeras exportadoras y que transfieren al gobierno), que en la actualidad está en un promedio de $35 \%$.

Son varios los factores que harían suponer que la tendencia por la expansión de la producción sojera en la región no se detendría:

1. Las demandas crecientes de la expansión demográfica en países que necesitan proteína vegetal para su transformación en proteína animal (como China e India).

2. La demanda de soja para la producción de biodiesel, que de no mediar restricciones paraarancelarias de la Unión Europea (UE) podrían convertir a Argentina en el productor de aproximadamente $10 \%$ de agrocombustibles, que ésta importa.

3. El cambio climático que tendencialmente provocaría el desplazamiento de cultivos como el maíz y el trigo. Esa superficie iría a soja, que sería uno de los cultivos menos afectados por el nuevo escenario (Barros, 2008).

4. Los costos. El agricultor argentino (incluyendo las retenciones, aún a precios relativos bajos de las commodities), obtiene más ingresos produciendo soja que cualquier otro cultivo alternativo contemporáneo a ésta (girasol, maíz) o leche o carne de manera extensiva (ganado a pasto). De ahí que la superficie implantada con el cultivo crece año tras año respecto a los demás cultivos (gráfica 1), que se mantienen estables, lo que implica que la soja está actuando como "cultivo colonizador" de nuevos territorios.

No obstante, la monocultura sojera afectará fuertemente los suelos, intensificará el uso de agroquímicos y fertilizantes y facilitará el desplazamiento de otras economías en la región, importantes para el desarrollo local como la producción láctea, la floricultura, la horticultura o la producción pecuaria.

La superficie sembrada con soja ha pasado de poco más de seis millones de hectáreas a mediados de los años noventa a 16,500,000 para la campaña 2008-2009, tal como se muestra en la gráfica anterior, con importantes transformaciones, incluso en 
Gráfica 1

Evolución de la superficie agrícola con soja, maíz, girasol y sorgo en Argentina (en hectáreas x 1000)

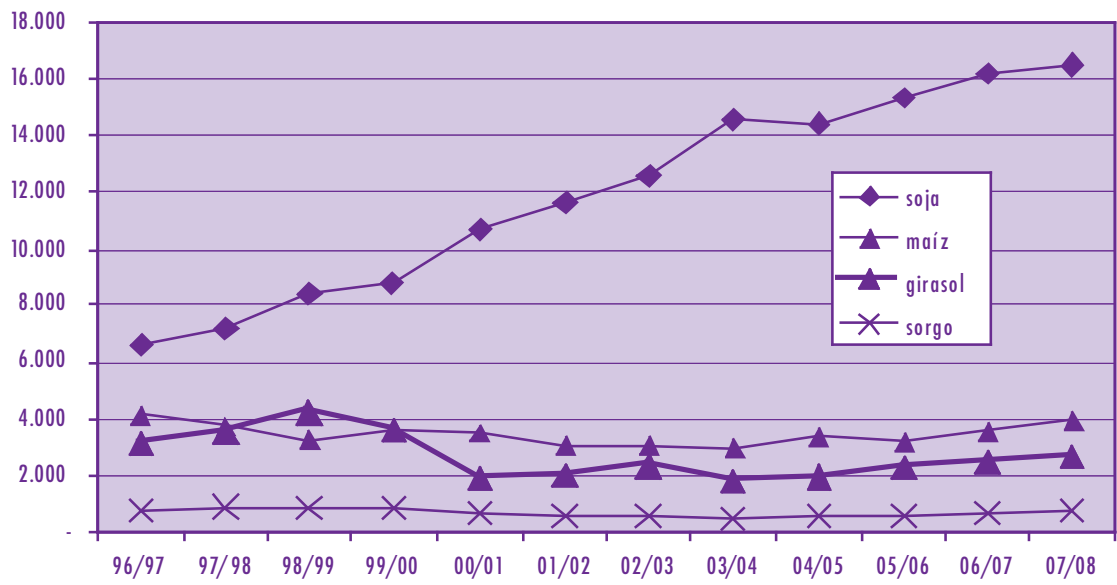

Fuente: Elaboración propia con base en datos de la Secretaría de Agricultura, Ganadería, Pesca y Alimentación de la Nación (2008).

algunos partidos (en algunos países se les llama departamentos) que tenían vocación pecuaria como Exaltación de la Cruz o General Rodríguez [provincia de Buenos Aires (u estado, según algunos países como México)] de la cuenca del río Luján y que ahora se están pasando a soja.

Mientras tanto la producción ha alcanzado 46 millones de toneladas (gráfica 2).

Gráfica 2

Evolución de la producción de soja argentina (en toneladas x 1000)

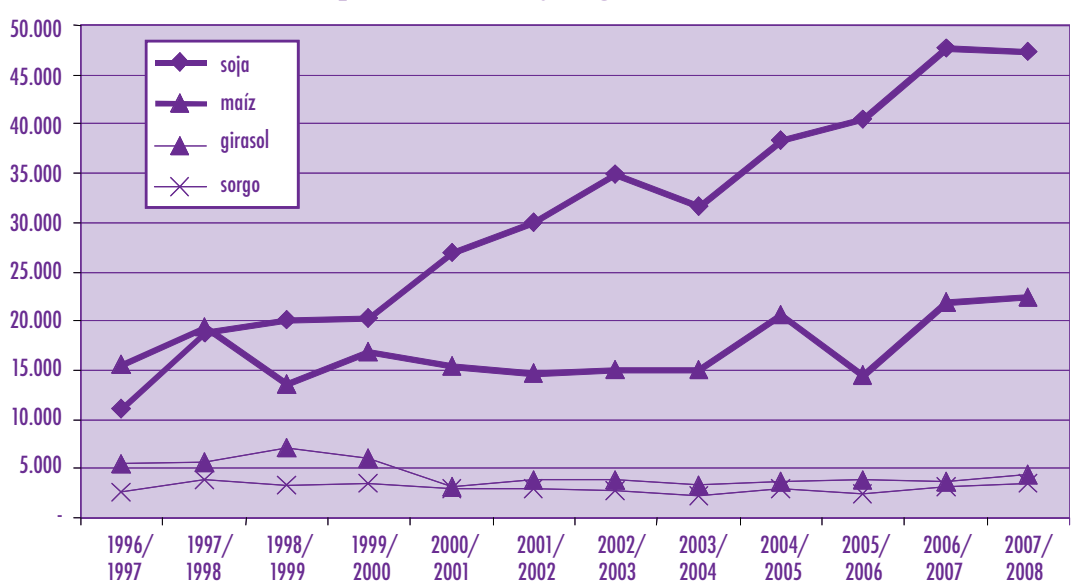

Fuente: Elaboración propia con base en datos de la Secretaría de Agricultura, Ganadería, Pesca y Alimentación, Argentina, 2008. 
El marco teórico de la economía ecológica: el agotamiento de los suelos y su explotación

La economía ecológica es la disciplina que se ocupa de la gestión de la sustentabilidad. En lugar de medir flujos e intercambios económicos, utiliza procesos energéticos y de materiales en términos biogeofísicos, para comprender el funcionamiento de una sociedad, su sistema económico y la demanda por sus recursos naturales. El “metabolismo social” (Martínez Alier, 2003; Toledo, 2008; Pengue, 2008) aborda el funcionamiento de los sistemas humanos por medio de la circulación de energía y materiales.

El análisis del flujo de materiales tiene en el estudio sobre las propiedades de los suelos y la circulación de los nutrientes un campo muy fértil de estudio en los análisis propuestos desde la economía ecológica (Pengue, 2006).

Muchas veces concepciones económicas equivocadas sobre las potencialidades de los suelos de Sudamérica llevaron a la sobreexplotación de los mismos y en otras tantas, aun conociendo sus limitaciones, lograron imponerse allí modelos de alta renta que agotaron el recurso rápidamente.

La economía convencional ha argumentado que el suelo - visto como un "recurso renovable", con ciertas condiciones - puede ser gestionado y por tanto aprovechado a perpetuidad. En realidad, en las condiciones de explotación actuales el suelo es un recurso agotable. El recurso de suelo fértil tiene un carácter desde el punto de vista biológico y químico vital; también es un recurso relativamente escaso y renovable sólo a una escala inalcanzable para la especie humana; es decir, un recurso que en la práctica resulta no renovable. Existe entonces una sustancial diferencia en cómo consideran la economía ecológica y la economía convencional los problemas ecológico-distributivos (Martínez Alier, 1995:143).

En general, los sistemas de monoproducción agrícola conllevan a una extracción selectiva de nutrientes del suelo, que lo agotan y fuerzan a una reposición vía fertilizantes minerales para recuperar la fertilidad actual, pero arrastran a crecientes niveles de contaminación y eutrofización, a la par de generar una mayor dependencia de importaciones del exterior.

La mayoría de los fertilizantes y agroquímicos consumidos en América Latina es importada. Para esta región, el principal límite para sus suelos reside en el estrés nutricional que pasa por la escasez o exceso de nutrientes (Pengue, 2005).

En la corta historia agroambiental argentina (de poco menos de 100 años) (Viglizzo y otros, 2002) es posible afirmar que la misma se ha visto acompañada por procesos productivos y manejos tecnológicos que a veces contribuyeron a degradar la 
base de recursos (Casas, 2001), pero en otros casos integraron, de forma más cercana a la sustentabilidad, sistemas productivos que supieron combinar de manera adecuada planteos rotacionales y prácticas integradas de manejo que si no elevaron, por lo menos sostuvieron la fertilidad.

Sin embargo, en las últimas décadas en el sur de América (las pampas en Argentina, el oriente en Bolivia, los cerrados en Brasil o los estados del este paraguayo) se está produciendo un desplazamiento importante y pérdida del sistema de rotaciones de ganadería extensiva por agricultura, para centrarse en cultivos de cereales y oleaginosas y en la producción intensiva del engorde a corral (feedlots). Ésta es la gran cuenca de transformación del sur de América, la cuenca del Plata.

Dentro de ella también hay grandes transformaciones en muchas de sus subcuencas. En la zona norte de la provincia de Buenos Aires, conocida como zona núcleo maicero (hoy convertido en sojero), el proceso aún es muy significativo. La zona noreste de Buenos Aires, que incluye a los partidos de la cuenca alta y media del río Luján, es otro ejemplo de esta transformación (los partidos de Ramallo, San Pedro, Baradero, Zárate, Exaltación de la Cruz, Luján, Suipacha, Mercedes, General Rodríguez, General Las Heras, Marcos Paz, Cañuelas, Navarro, Lobos, Roque Pérez, Monte y San Andrés de Giles integran la cuenca en su totalidad o los representan en porcentajes importantes).

La zona se encuentra dividida en dos grandes subzonas: una donde predominan los sistemas ganaderos y otra donde sobresalen los sistemas frutihortícola y florícola. En la subzona de sistemas ganaderos $67.4 \%$ de los suelos tiene aptitud predominantemente ganadera, $23.5 \%$ registra aptitud agrícola ganadera y sólo el $2.5 \%$ restante de la superficie posee aptitud agrícola. No obstante lo indicado, el cultivo de soja se está instalando en la región a pesar de esta relativa cualidad del suelo.

El proceso ha llevado a un evidente síndrome de sustentabilidad, el de agriculturización, que en el caso comentado ya puede llamarse de sojización, con características propias en los ámbitos global, nacional y regional.

Este cambio en el modelo productivo produjo transformaciones en los agroecosistemas de la región pampeana, cuyas consecuencias fundamentales han tenido relación con los procesos de erosión y pérdida de fertilidad manifestados en las principales cuencas productivas de la región. Junto con la siembra directa, el consumo de fertilizantes ha sido uno de los factores representativos de la década de los noventa.

Desde la implantación de la siembra directa, el consumo de urea y fosfato diamónico han sido de los fertilizantes que comienzan a consumirse más (Darwich, 2002).

Vol. 40, núm. 157, abril-junio / 2009

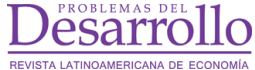


Indicadores biofísicos de (in)sustentabilidad: pérdida y exportación de nutrientes

Desde mediados de la década de los setenta los suelos de la región pampeana comienzan a sufrir una extraordinaria presión, fruto de la transformación de la actividad agrícola generada por la adopción de la moderna tecnología, la concentración económica y el aumento de la escala, las nuevas formas organizativas y la fuerte orientación y dependencia del mercado exportador.

En ese periodo comienza una veloz expansión hacia las monoculturas maicera, girasolera y triguera (agriculturización) para más adelante concentrarse en el cultivo de soja (sojización), práctica que se difundió velozmente en las épocas posteriores, al ser combinado en un planteo rotacional, bajo el sistema de siembra directa, con trigo.

Esta situación mejoraba la condición financiera de los agricultores, al facilitar la combinación ajustada de ciclos productivos y, por ende, encajar tres cosechas cada dos años. En una primera etapa, la intensificación agrícola se llevó adelante basada en labranzas convencionales que incrementaron los procesos de erosión hídrica y eólica y luego con la puesta en marcha desde mediados de los noventa con la técnica conservacionista de la siembra directa (Pengue, 2000:102).

La actividad agrícola forma parte de cada uno de los ciclos de los nutrientes. De hecho la fijación en el suelo, su extracción, circulación y sustitución al mismo funcionará de distinta manera, en tanto y en cuanto los distintos productos de las transformaciones agropecuarias se transformen y consuman en fuentes demandantes cercanas o lejanas del lugar original donde se encuentra el nutriente.

Entonces, no será lo mismo revisar lo que sucede en la producción del maíz, girasol, el trigo o la carne (vacuna) que con la soja, en especial cuando esta última es destinada casi en su totalidad a los mercados externos, mientras los primeros mantienen - con sus vaivenes - un consumo interno algo sostenido, desigual, pero integrado aun en parte hacia la sociedad local.

El cultivo de soja ha desplazado a otros cultivos, algunos de ellos vinculados a la demanda nacional de alimentos y, por ende, mucho más relacionados a la soberanía alimentaria de los argentinos.

En la actualidad la soja ocupa más de $50 \%$ de la superficie total con agricultura en Argentina. En los últimos cinco años, además del avance sobre frontera agropecuaria, la soja alcanzó a desplazar alrededor de 4,600,000 hectáreas dedicadas a otras producciones (maíz, algodón, girasol, fruticultura, horticultura y pasturas para ganadería) (Pengue, 2006:37).

Si bien las técnicas agronómicas cambiaron a lo largo de los últimos 15 años (nuevas variedades, fechas de siembra, sistemas de labranza y manejo, control de malezas 
y enfermedades, barbecho químico, siembra directa), es posible adelantar que en la cuestión de fertilizantes minerales el consumo en el cultivo de soja ha sido escaso, lo que implica que existe una exportación neta de diferentes nutrientes.

Sin embargo, la pérdida de nutrientes del suelo no se debe sólo a la extracción que hacen las cosechas, sino que el manejo que se haga del mismo y los procesos erosivos al igual que la lixiviación tienen un papel importante dentro de este flujo de materiales.

La alta extracción de nutrientes, las reducidas prácticas de manejo y su concentración en pocos cultivos sin ganadería y la muy escasa reposición han dado como resultado en la degradación de los suelos, sobre todo aquéllos con mayor frecuencia de soja en la rotación, es decir, aquellos suelos que han seguido un modelo de agricultura continua durante muchos años.

Por otro lado, es importante considerar que a diferencia de otros cultivos, la soja se "produce" incluso en suelos ya degradados o con bajo contenido general de nutrientes, lo que puede solapar el proceso de degradación por varios años.

Hasta el momento, a pesar de ser pareja la extracción de nutrientes, la gran disponibilidad en el suelo de algunos de ellos enmascara un posible déficit.

A diferencia de lo que ya se percibe con el nitrógeno, el fósforo, el azufre, la aún importante disponibilidad de nutrientes como el $\mathrm{K}$, el Ca, el $\mathrm{Mg}$ o el S, no se revisa muchas veces con la misma intensidad, a pesar de ser elementos que de manera recurrente también salen junto con las cosechas (gráfica 3).

Gráfica 3

Pérdidas de nutrientes respecto a su condición original en un suelo Argiudol típico, Serie Pergamino, después de 80 años de agricultura continua

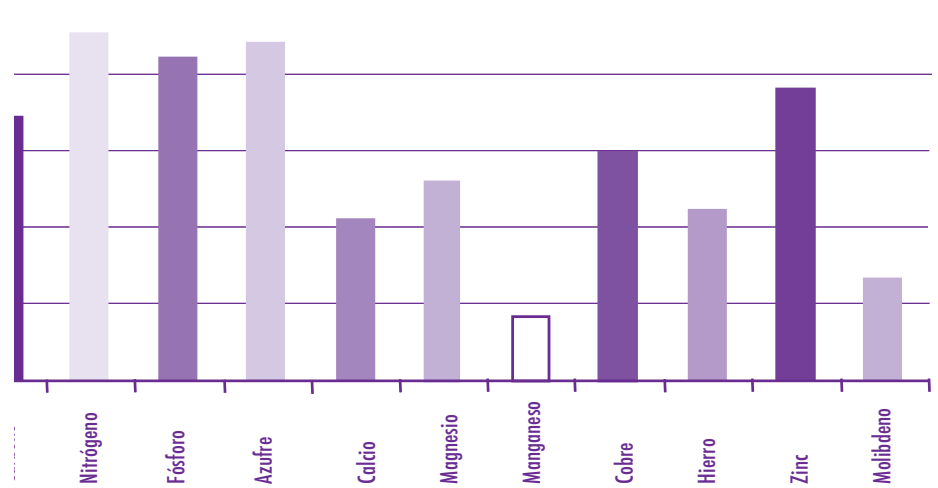

Fuente: Andriulo et al.,1996. 
Por otro lado, la alta producción de soja esconde una elevada extracción de los nutrientes del suelo, al exportarse casi $90 \%$ de la soja cosechada (ya sea directamente en granos o transformada en tortas y aceites).

Si la comparamos con otros cultivos como el maíz, el trigo, el girasol o la alfalfa, la soja es uno de los cultivos que más nutrientes extrae del suelo por unidad de materia seca producida. Éstos los obtiene de dos formas: por una eficiente extracción selectiva del suelo y producción propia (caso del nitrógeno) o por un agregado continuo externo vía fertilizantes minerales.

La soja provee un rastrojo rico en nitrógeno (baja relación carbono nitrógeno) que se descompone rápidamente, dejando al suelo con muy poca cobertura y expuesto a la acción erosiva. Es decir, incluso con siembra directa, pero sembrando soja sobre soja, la práctica agronómica es igualmente insustentable. Allí aparece una doble vía de reducción del stock de materia orgánica del suelo. Por un lado, porque la tasa de adición de rastrojos no alcanza a compensar la tasa de mineralización y, por otro, porque la erosión se lleva aproximadamente $0.1 \%$ de materia orgánica por cada centímetro de suelo perdido, de acuerdo con mediciones realizadas por el Instituto de Suelos del INTA (el Instituto Nacional de Tecnología Agropecuaria, de Argentina) en la subregión Pampa ondulada.

El efecto de la agricultura continua, especialmente sojera, no sólo muestra el desbalance que se produce en el sistema por este desplazamiento en términos de nutrientes perdidos, nitrógeno y fósforo, sino que se produce un incremento sustantivo en los consumos de energía fósil, contaminación con pesticidas, disminución del carbono intercambiable, aumento de los riesgos de erosión e intervención del hábitat.

La salida de nutrientes junto con la soja argentina muestra un fuerte punto de inflexión desde mediados de los años noventa (Pengue, 2006). No es una cuestión menor que en 1996 se libere comercialmente la soja transgénica, que los agricultores adoptan de manera masiva en muy poco tiempo. El fuerte pico de extracción de nutrientes comienza a mostrarse allí acompañando un desplazamiento hacia la monocultura sojera (gráfica 4).

\section{El caso del fósforo y su agotamiento regional}

Organismos públicos y entidades privadas (INPOFOS, CREA y AAPRESID) han venido analizando los niveles de fósforo disponible en el suelo, sobre todo durante la década de los noventa mediante muestreos de más de 1,200 tomas permanentes en las provincias de Buenos Aires, Entre Ríos, centro y sur de Santa Fe, este de Córdoba y este de La Pampa. En la actualidad toda la provincia de Entre Ríos, el este y sureste de la 
de Buenos Aires poseen suelos predominantemente deficientes en fósforo (menos de 10 ppm). Esta situación es similar en el sur de Buenos Aires (Pengue, 2006:219).

Una conclusión general es que los niveles de fósforo han disminuido en zonas de la región pampeana originalmente bien provista de este elemento (Viglizzo y otros, 2002; Casas, 2003). La frontera entre las áreas de suficiencia y deficiencia, 20 y $10 \mathrm{ppm}$ respectivamente, se desplaza en forma paulatina hacia el oeste. Lo mismo sucede en el sur de Santa Fe y el oeste de la provincia de Buenos Aires. Los suelos agrícolas del noreste santafesino presentan bajo contenido de $\mathrm{P}$ disponible al igual que porciones importantes del territorio del suroeste cordobés.

La tasa de extracción anual está creciendo en toda el área a su vez, por el cambio importante hacia agricultura continua. Se puede observar que la extracción del nutriente (poco móvil en el suelo) se concentra en las áreas del núcleo sojero/maicero de Argentina, extendiéndose incluso en la actualidad hacia las zonas más marginales del sector productivo donde también se comienza a producir soja.

Sin embargo, hoy la externalidad negativa derivada de la exportación de los nutrientes en los granos no es considerada. En el caso de la soja y el fósforo se altera su ciclo biogeoquímico regional (debería considerarse al fósforo un recurso no renovable), al desplazarse el nutriente a miles de kilómetros de sus lugares naturales (Pengue, 2004).

Por una parte Argentina exporta granos con una extracción importante de nutrientes, incluso bajo sistemas de labranza diferentes hasta con aquellos "más sustentables" como la siembra directa, incrementando la deuda ecológica regional al no permitir la reposición natural rotacional y, por otro lado, importa cargamentos con fertilizantes minerales para suplir y mantener artificialmente estos niveles productivos. En ninguno de los dos casos esta extracción-reposición se puede contabilizar como un crédito al balance final de nutrientes del suelo, lo que implica un costo directo no reconocido y por tanto una externalidad pagada socialmente por la degradación del recurso natural. Pero además esta artificialización y utilización del sistema —en especial cuando se aplican fertilizantes minerales - esconden efectos degradatorios del ambiente (eutrofización de los cursos de agua o contaminación de las napas).

\section{La deuda ambiental por pérdida de nutrientes}

En la teoría económica, la doctrina sobre las bondades de la libertad de comercio lleva el nombre de teoría de las ventajas comparadas (Martínez Alier, 1998:143). Sin embargo, desde esta visión poco caso se hace sobre las formas de extracción y degradación, incluso de los recursos que hacen a ese crecimiento económico. Como he 
argumentado, Argentina ha exportado y exporta millones de toneladas de nutrientes naturales que, por supuesto, no se recuperan de manera racional.

Extracción de nutrientes, degradación, erosión y desertificación tienen una consecuencia ambiental directa, apenas perceptible hasta su materialización en la imposibilidad productiva, lo que se manifiesta en algo aún más terrible: el aumento de la pobreza, la devaluación económica de los recursos y el aumento del costo social.

En el pasado Argentina tuvo sobre los suelos pampeanos un proceso de descarga y reposición que en su historia agrícola centenaria le permitió de alguna forma mantener su base de nutrientes, en un proceso de recuperación natural. En algunos casos, y por cierto desde los orígenes de la agricultura, estos procesos tendieron a disminuir, pero nunca hasta ahora se puso en riesgo la base productiva. En la actualidad, un nuevo proceso de intensificación de la agricultura, de base sojera, produjo una extracción selectiva de nutrientes con escasa posibilidad de recuperación natural.

El caso de la agricultura sojera industrial es particular. La recuperación de los nutrientes no se ha realizado, como ya se mencionó, por la vía natural ni tampoco por la vía de la reposición mineral, estando aún los consumos muy alejados de la demanda potencial de cada cultivo. Por tanto, es más que claro que la riqueza exportada proviene directamente del suelo pampeano.

La historia de los cultivos en las pampas se desarrolló sin el agregado de fertilizantes minerales. La llegada de estos fertilizantes nitrogenados y fosforados a los planteos de cereales y, en menor medida, de oleaginosas ha adquirido sólo recientemente (última década) una dimensión importante, lo que ha estado asociado más a la posibilidad de aumentar los rendimientos de los cultivos que a una conciencia sobre la necesidad de reposición de nutrientes del sistema para conservar el capital natural.

En el caso de la soja, otro proceso que será más que importante relevar es la exportación del modelo pampeano (síndrome de la pampeanización) hacia otras zonas marginales (Pengue, 2005b) para la producción de granos como en el caso del Chaco. En la actualidad, en Argentina se está avanzando sobre áreas ricas en biodiversidad, que hoy representan 90\% de la frontera agropecuaria (Pengue, 2009), y que están generando un "desbosque masivo" por arriba y por debajo, produciendo una rápida degradación de la estructura y la base de nutrientes de suelos más frágiles.

Los efectos de la imposición del modelo pampeano en otras ecorregiones que no son pampa tienen también impactos importantes no sólo en la deforestación, sino a su vez sobre la biodiversidad. La aparición de malezas tolerantes (más de 15) (Pengue, 2005) y resistentes, como el sorgo de Alepo (Sorghum halepense), al herbicida gli- 
fosato (Service, 2007; Pengue, 2009; Binimilis, Pengue y Monterroso, 2009) alertan sobre un cuadro de crisis en el control de las malezas, como resultado de esta bioinvasión, que ni los Estados ni las empresas están tomando con la debida consideración y dedicación.

Este nuevo síndrome, por la imposición de un paquete tecnológico completo, incluso mucho más agresivo en términos de los insumos externos que se están aplicando (herbicidas, insecticidas, fertilizantes), lo he denominado pampeanización (Pengue, 2005, 2008) y tiene características propias (cuadro 1).

Este síndrome de pampeanización en el Chaco genera un proceso de degradación doble del suelo, al desmontar por arriba y exponer a las fuerzas del riguroso clima chaqueño (altas temperaturas, escasa humedad, erosión eólica) y degradar por debajo una frágil estructura de un suelo, que si bien es productivo, debe ser manejado con prácticas de agroforestería, donde el bosque, y no la agricultura, sea su vocación primera.

$\mathrm{Al}$ igual que los nutrientes en los granos, la exportación de agua virtual (Pengue, 2006c) formará parte de las discusiones en el uso sustentable de nuestros recursos y en el cálculo de las deudas ecológicas de los países importadores de nuestros granos.

Cuadro 1

Síndrome de (in)sustentabilidad

Pampenización

Precio de la tierra

Nivel global

Concentración de las cadenas de comercialización y aldea global

Eficiencia productiva y traslado de costos norte-sur

Falta de política agropecuaria y ambiental

Nivel nacional

Desconocimiento y falta de evaluación de impactos

Escaso ordenamiento del territorio

Escaso trabajo interprovincial para el desarrollo regional

Desnaturalización del concepto de economía regiona

Corrupción

Falta de conocimiento y movilidad social y políitca

Dominio conceptual del modelo tecnológico

Nivel regional o agroecosistémico

Devaluación de la agricultura regional o local

Falta de incentivos en el nivel de finca para el trabajo de mediano plazo

Nuevo actor rural "moderno" y desaliento al productor y al agricultor

Fuente: Pengue, 2005:150; 2008.

Vol. 40, núm. 157, abril-junio / 2009

Desartoris 


\section{Metodología y análisis de resultados} económicos de la exportación de nutrientes

Las exportaciones de nutrientes generados por el modelo sojero no pueden esconder a los ojos de la economía ambiental una salida creciente de los mismos que no dejan de poner en duda la supuesta eficiencia de la agricultura exportadora. En la región pampeana, la externalidad generada por la extracción de nutrientes ha sido evaluada por varios autores (Flores y Sarandón, 2002, Pengue, 2006; Pengue 2006b), pero respecto a la exportación de nutrientes, sólo los de mayor demanda por los cultivos en términos de volumen requerido o que limitaban los rendimientos, al ya no disponerse con ellos en el suelo.

Éstos son nitrógeno, fósforo y potasio. En este sentido, las dosis minerales aportadas en promedio por hectárea durante la última década no estuvieron asociadas a las tasas de extracción de los principales cultivos (trigo, maíz y soja), lo que significa una pérdida de la "caja de ahorros" del suelo.

En términos del volumen extraído con el cultivo de soja, desde los comienzos de la agriculturización en la década de los setenta (1970-1971) hasta 2005, Argentina ha perdido 11,354,292 millones de toneladas de nitrógeno (ya descontada la reposición natural), 2,543,339 millones de toneladas de fósforo y valores muy elevados de los demás nutrientes y oligoelementos, a pesar, como he comentado, de su buena disponibilidad en un suelo, que no obstante se va vaciando (Pengue, 2006b).

A valores en dólares, ${ }^{1}$ y sólo tomando como referencia una equivalencia con la restitución de lo perdido (que asumimos es una simplificación de la realidad del balance de nutrientes), con fertilizantes minerales, los costos incumben cifras sumamente elevadas. Argentina debería ser resarcida con 2,895,344,460, 2,638,055,818, $890,168,650,461,509,880,86,251,130$ y 71,531,320 dólares para el nitrógeno, potasio, fósforo, azufre, calcio y magnesio exportados, respectivamente (Pengue, 2006b).

1 Los valores utilizados han sido 255 dólares por tonelada para la urea perlada, 350 dólares de fosfato diamónico, 38 dólares de $\mathrm{KO}_{2}$, 70 dólares de dolomita, 260 dólares de sulfato de amonio. Los datos referenciados se tomaron del programa FERTILIZAR del INTA y la Secretaría de Agricultura, Ganadería y Pesca de Argentina en conjunto con empresas privadas. Se consideró asimismo que se utilizaría este valor, equiparando al fertilizante incorporado respecto al exportado en una relación 1:1. Los fertilizantes minerales no aportan en tanto en esa relación, dado que su contenido específico del nutriente es menor, por ejemplo en el caso de la urea de $46 \%$, fosfato diamónico, $64 \%$ y sulfato de amonio, $45 \%$. 
En el caso de la producción sojera pampeana, la extracción de nutrientes ha sido especialmente importante, por ser ésta una de las áreas de mayor producción de la oleaginosa.

Las provincias pampeanas (Buenos Aires, Santa Fe y Córdoba) alcanzaron en conjunto una extracción total de elementos mayores (N, P, K, Ca, Mg, S) de 20,305,794 toneladas y 244,449,822 kilogramos de micronutrientes (B, Cl, Cu, Fe, Mn, Mo, Zn), siempre tratando exclusivamente la extracción por la soja.

La extracción por hectárea durante todo el periodo ha sido también muy alta, llegando a los 158 kilogramos para los nutrientes principales (N, P, K, Ca, Mg, S).

La intensificación de la agricultura sojera, vinculada a las exportaciones y a la dependencia de los recursos obtenidos de las retenciones (impuesto del gobierno a la exportación de granos que llegan a 35\%), hace que este proceso de producción permita al gobierno contar con divisas para el pago de deuda y mantener un sistema de planes sociales, que hoy día ya se debía haber superado, con propuestas productivas y de trabajo superadoras, utilizando los mismos recursos de estos impuestos. Para un plan nacional de desarrollo rural, la cifra retenida no es menor, al estar hablando de prácticamente 3,000 millones de dólares anuales por esta vía, que se pierden en una administración social ineficiente y no vuelven, como es posible hacer, al reproducirse en producción y trabajo hacia el propio sector rural, hoy amenazado por esta agricultura industrial que por otro lado se fomenta.

A medida que se avanzó en el proceso de agriculturización, considerando los tres cultivos, trigo, soja y maíz, las pérdidas de nutrientes y el costo de reposición de los mismos por restitución mineral ha ido en aumento. Ello se produce por dos motivos: por el aumento de la superficie ocupada por los principales cultivos y por la demanda de cada vez más nutrientes, al utilizarse especialmente en este periodo nueva genética que incrementa los rendimientos, pero demanda una tasa extractiva mucho mayor (en el campo se dice "hay que darles de comer, para que rindan..."). Incluso el aporte de fertilizantes minerales se rige por la relación beneficio-costo por unidad producida, pero no en términos de la necesaria reposición natural, cuyo "capital" se degrada de manera casi constante.

\section{Deuda externa y deuda ecológica por pago con nutrientes naturales}

Muchas veces hemos escuchado que hay que incrementar la producción para generar crecientes saldos exportables, que nos permitan hacer frente a los pagos de los servicios de la deuda externa. No obstante, poco se ha revisado respecto a la salida de nutrientes de los suelos pampeanos e incluso su posible valoración como hemos mostrado, incorporando este valor a los precios que realmente se están manejando. 
La incorporación del costo de los nutrientes naturales exportados junto con el agua virtual utilizada debería formar parte de instancias de análisis, sobre todo cuando se trata de una degradación, que para ser recuperada por los mismos canales de la intensificación productiva sólo se haría por medio de nutrientes minerales, que tienen un importante costo en el mercado internacional.

En el caso de los macronutrientes, la cifra que se debería haber restituido al ambiente para reponerlos desde el momento en que la soja llega a la región alcanza un promedio anual de alrededor de 400 millones de dólares, por ejemplo para el último quinquenio.

También es importante tener en cuenta que a pesar de lo demostrado respecto a la buena disponibilidad general de micronutrientes en las pampas, su extracción es creciente y estos factores - por ser en el futuro su posible deficiencia - son importantes limitantes para la productividad de las plantas. Su salida recurrente en algún tiempo obligará a la utilización de fertilizantes minerales, que en estos casos son mezclas especiales, cuyos costos son proporcionalmente más altos que los de los macronutrientes.

Así como no se incluye el valor de los nutrientes incorporados y el agua utilizada para su producción en los precios internacionales de la soja circulante, el efecto de los subsidios de los países industrializados toma en cuenta otra situación que pasa por la subvaluación de los productos exportados por las economías en desarrollo, al colaborar en la depresión de los precios internacionales de estas producciones.

Ello impulsa a una mayor intensificación de la producción en los países del sur, para, según el argumento, continuar siendo competitivos en estos distorsionados mercados internacionales.

\section{El caso de la cuenca del río Luján}

Por su proximidad a la ciudad más importante de Argentina, Buenos Aires, y por las transformaciones que se están produciendo en la agricultura de este país, el caso de la cuenca del río Luján (Dichón y otros 1999:1) es un interesante escenario de la intensificación de las transformaciones en el sector rural, que incluso están desplazando a otras economías productivas para concentrarse básicamente en cultivos de exportación, como la soja, por su propia cercanía y salida por los puertos, disminución de los costos de fletes (largo y corto), disponibilidad rápida de maquinaria agrícola, equipos y asesoramiento técnico.

La cuenca del río Luján es un territorio sin limitaciones físicas serias para la producción agrícola (mapa 1) ni situaciones climáticas importantes. Está ubicada hacia 


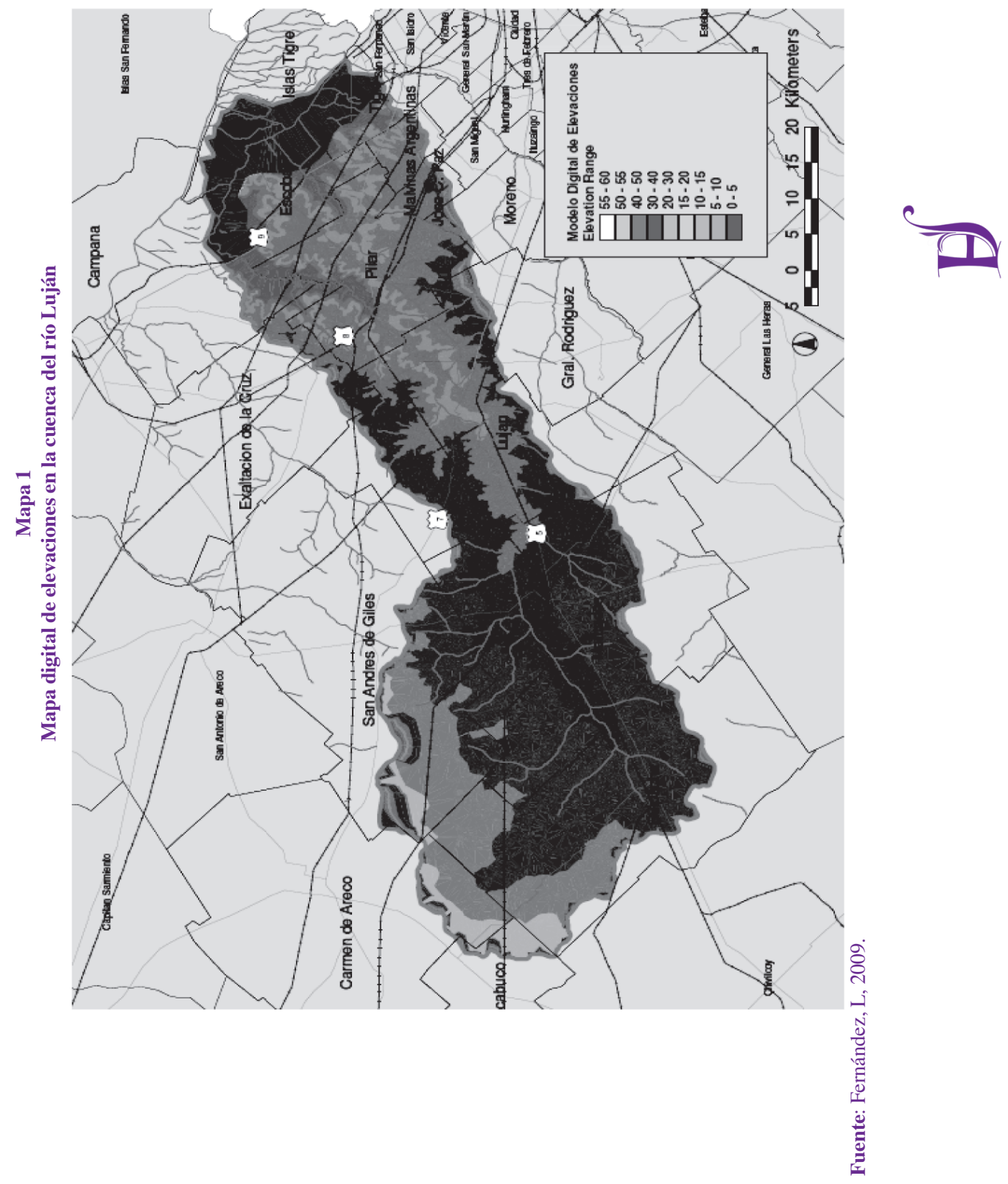

Vol. 40, núm. 157, abril-junio / 2009

Desaarrollo 
el noreste de la provincia de Buenos Aires y abarca unos 3,000 km ${ }_{2}$. El cauce principal recorre $128 \mathrm{~km}$ en sentido suroeste-noreste desde su nacimiento en el partido de Suipacha (como arroyo Los Leones) hasta su encuentro con el delta del Paraná. Atraviesa nueve partidos (Chivilcoy, Mercedes, Luján, Pilar, Exaltación de la Cruz, Campana, Escobar, Tigre y San Fernando) y a lo largo del primer tramo mencionado recibe tributarios también desde los territorios de San Andrés de Giles y General Rodríguez. La población del área es de aproximadamente 1,000,000 de habitantes.

La zona ha sido utilizada inicialmente para explotación agrícola y ganadera extensiva, sin embargo con el crecimiento de las ciudades se ha incrementado también la actividad industrial, sobre todo en el curso inferior. En la actualidad el sector agrícola está teniendo una intensa reorientación hacia la producción de soja.

No obstante, según varios autores, los estados del recurso hídrico y del ambiente general de la cuenca - en particular en las cuenca alta y media - irían de buenos a muy buenos (Momo y otros, mimeo, s/f).

Además, el crecimiento territorial que viene teniendo el cultivo de soja en la región (gráfica 4) y su paquete asociado de siembra directa y glifosato, fertilizantes

Gráfica 4

Evolución de la superficie implantada con soja en las provincias de la cuenca del río Luján (elaborado por Zuberman, F. 2009)

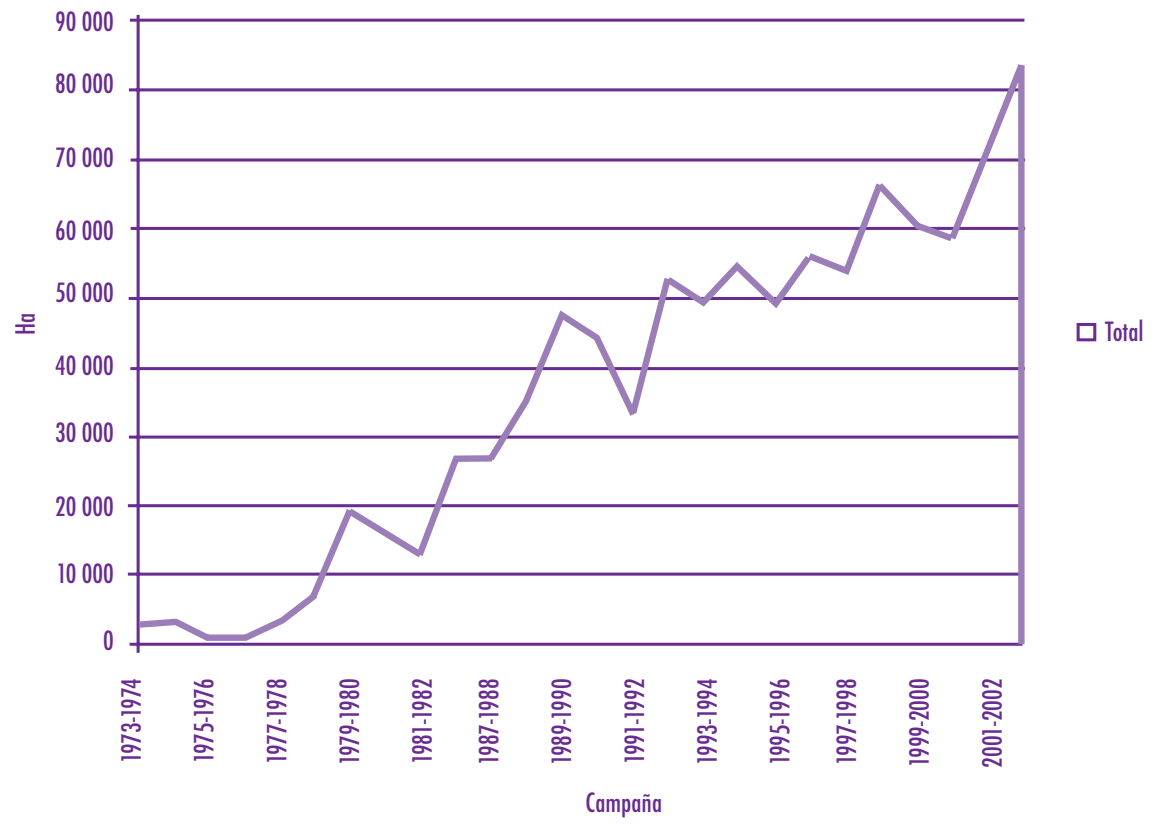

Fuente: Secretaría de Agricultura, Ganadería, Pesca y Alimentación. www.sagpya.mecon.gov.ar. 
y agroquímicos en general, permitirían predecir un aumento del riesgo ambiental relativo para toda la región, con base en la metodología desarrollada por Viglizzo y otros (Viglizzo y otros, 2002).

La zona que más rápidamente ha crecido en superficie implantada respecto a los nueve partidos de la cuenca es General Rodríguez, que pasó de 300 hectáreas sembradas en 1978 a 11,000 en la campaña 2007-2008.

A diferencia de lo que sucede en otros escenarios netamente rurales de Argentina, en el caso de la cuenca del río Luján también existe una fuerte expansión de los sectores urbanos y periurbanos. En este sentido, hay una preocupación manifiesta por amplios y crecientes sectores sociales, en particular de los que se encuentran en las áreas de borde de los cultivos, por los efectos nocivos para la salud y el ambiente y por la expansión del cultivo de soja en áreas colindantes con sus viviendas permanentes.

La situación ya inocultable de crecientes casos vinculados al aumento de enfermedades posiblemente derivadas de los procesos de intensificación agrícola en Argentina (casos del barrio Ituzaingó, que generaron, por ejemplo, la intervención presidencial) está poniendo una voz de alerta referida a la urgente necesidad de revisar el crecimiento de estos sistemas productivos en estas áreas tan cercanas a la población local. En la misma cuenca comienzan a haber denuncias referidas a fumigaciones con glifosato (Noticias Mercedinas, 2009) en Mercedes, Gowland, García, Alberdi y otras localidades.

La extracción de nutrientes - en particular con un cultivo que prácticamente se ha concentrado en el área junto con la salida de agua virtual - es notoria, pues la región ya exporta en los últimos guarismos poco más de 300,000 toneladas.

En los partidos de la cuenca alta y media, incluso los tambos y la ganadería, se está cediendo terreno a la soja, lo que promueve la transformación de las tradicionales rotaciones agrícolas ganaderas a una agricultura continua.

Los procesos de agriculturización (sojización) y el aumento de la ganaderización concentrada (feedlots) se confirman en la cuenca, no así la pampeanización, en tanto que toda el área ya estaba capitalizada y en manos de agricultores con cultura pampeana (agricultura y ganadería extensiva).

El caso de los procesos de feedlots deberá revisarse con mucha más amplitud, en tanto que el cambio de los sistemas de producción de ganado en Argentina - de animales que pastaban libremente en territorios extensivos a su concentración (derivada del alto precio de la tierra que se dedica a agricultura) - en pequeños espacios para la producción están generando algunas consecuencias negativas. Varias localidades bonaerenses, ubicadas en la franja agropecuaria de la cuenca y en otras áreas del país, 
han comenzado a padecer problemas ambientales por la cría intensiva en espacios reducidos de vacunos y aves. Invasión de moscas y roedores, olores nauseabundos y hasta polución en las napas son algunas de las consecuencias de estas prácticas. La falta de legislación que regule el engorde y el escaso control estatal sobre los establecimientos hacen que el fenómeno no sólo no se detenga, sino que siga aumentando (Vulcano, 2006). Saladillo, Luján, San Andrés de Giles, Pilar, Exaltación de la Cruz y Pergamino son algunas de las zonas donde se han registrado problemas con la instalación de los procesos de feedlots, que concentran a 500 vacunos por corral, toman 50 litros de agua por día y producen 40 kilogramos de excretas diarios por animal, muchas veces con un tratamiento sólo superficial. En la localidad de Saladillo, hay 16 feedlots. Lo mismo comienza a suceder en Luján y en Exaltación de la Cruz. Argentina cuenta con 1,300 feedlots inscritos, por lo que el problema ambiental y la salud de hecho pueden ser un escenario de creciente conflictividad. Se estiman 1,500,000 cabezas de ganado en feedlot y un apoyo incluso creciente de parte del gobierno para intensificar este tipo de producciones, que se destinan en su totalidad al consumo interno. La política pública en este sentido, como lo fuera previamente con el caso de la soja, estaría definida. Se vincula directamente a los objetivos de incrementar la producción, sin consideración de sus externalidades. Incluso a finales de 2008 se estaban proyectando cinco nuevos feedlots para 40,000 cabezas de ganado cada uno, que serían destinados al engorde del ganado overo (el ternero de la vaca lechera) y orientados a la exportación y el consumo interno (p. 12, 2008).

La problemática no sólo arrastra nuevos problemas de estética o superficiales, sino que concatena un crecimiento y aparición de riesgos por posibles enfermedades transmitidas por roedores o moscas que se desarrollan tanto en el feedlot como en los criaderos de pollos.

Otro problema serio producido por las intensificaciones agrícola y pecuaria es la contaminación por nitratos, cuyos datos están relevándose en proyectos específicos como el del estado del recurso hídrico en la cuenca del río Luján.

\section{Conclusiones}

La globalización del sistema mundial de alimentos conlleva a una sobreexplotación importante de recursos y a una aceleración de los ciclos productivos y alteración de los ciclos biogeoquímicos, en términos no sustentables, lo que genera pasivos ambientales crecientes.

Existen nuevos procesos de regionalización mundial, riesgosos no sólo en términos comerciales, sino en cuanto a la nueva distribución y apropiación de los recursos usados. 
Es así que la nueva distribución del poder económico viene aparejada de un cambio en la distribución geográfica de los impactos sobre el ambiente. Si desde el punto de vista ecológico se define al poder como la capacidad de internalizar ventajas ambientales y externalizar los costos ambientales, bien puede suponerse que el alargamiento de las cadenas económicas dé origen a un proceso de concentración de las ventajas en los extremos superior y las desventajas en el extremo inferior.

En otras palabras, los costos ambientales en que incurren las cadenas transnacionales de creación de plusvalía serán especialmente altos en los países del sur y del este, mientras que las economías post-industriales irán tornándose cada vez más benignas y afines con el ambiente.

Entonces el caso de la agricultura regional se muestra paradigmático. Los nuevos espacios "vacíos" de producción comienzan a ocuparse y sobre ellos se avanza sin una consideración sostenible en el uso del recurso y tampoco se incluyen los costos de transformación involucrados.

La pérdida de biodiversidad es un proceso también intenso y en algunos países está afectando recursos directamente vinculados a las ricas zonas boscosas. En el caso de Argentina, se avanza en el cultivo de soja sobre campos ya transformados (provincias de Buenos Aires, Santa Fe y Córdoba), pero también de la mano de las nuevas variedades se impulsa un proceso de sojización hacia el caldenal pampeano (Prosopis caldenia), los bosques de ñandubay entrerriano, las provincias de Corrientes, Misiones, el NEA con las ecorregiones del Chaco y el Monte y hasta parte del NOA en las selvas pedemontanas de Yungas.

Los problemas vinculados a la pérdida de biodiversidad se reflejan en el éxito de bioinvasiones como el de los nuevos biotipos de sorgo de alepo resistente al glifosato (SARG), que ya prácticamente han sido identificados hasta en la región pampeana.

Los cálculos que desde la economía ambiental pueden lograrse para una mínima valuación de las externalidades involucradas en los sistemas de producción de monocultivo como la soja no pueden incluir adecuadamente los costos por los efectos producidos sobre la biodiversidad local y regional, la pérdida "completa" de los nutrientes, los costos por problemas de estructura o el aumento tendencial en los niveles de riesgo por contaminación al incrementarse los niveles de agroquímicos (fertilizantes, insecticidas, fungicidas y herbicidas) utilizados en la producción.

El crecimiento de los niveles de producción y el aparente enriquecimiento de ciertos sectores de la economía global no pueden soslayar los impactos que los procesos de transformación de los recursos tienen o tendrán en la sostenibilidad, incluso débil, de todo el sistema. 
La pérdida de nutrientes es parte de este costo, la pérdida o mejora de los contenidos de materia orgánica, los problemas de acidez o alcalinidad, la pérdida de estructura, los problemas de infiltración o arrastre del agua en el suelo, las tasas de erosión y el riego, son también otros factores que no están incluidos en los costos.

La agricultura industrial está generando importantes y profundas consecuencias sociales a escala del país. El modelo no puede ocultar la deuda interna en términos de la disminución de la mano de obra en el sector primario, ni la consiguiente especialización y demanda de calificación técnica para los puestos disponibles, ni la pérdida de la cultura rural, ni el vaciamiento del campo, ni la concentración en grandes empresas transnacionales y un constante aumento de la unidad de la escala económica. Tampoco puede mirar de soslayo la cuestión socioambiental. Argentina enfrenta serios problemas derivados de la llegada de nuevas enfermedades, que deberían haberse previsto si el enfoque frente a las transformaciones producidas por la intensificación y sus vinculaciones a los incipientes procesos de cambio climático hubiera sido preparado desde hace más de 10 años atrás, con uno que fuera del tipo holístico.

El modelo global de agricultura industrial que ha llegado a Argentina en la última década encontró una estructura agroproductiva perceptiva a los cambios, que adoptó en términos técnicos muy rápidamente, nuevos paquetes tecnológicos como la siembra directa, las sojas, maíces y algodones transgénicos, la intensificación en el uso de agroquímicos y nuevas maquinarias especializadas. Se abandonó prácticamente la agricultura familiar a su suerte y ahora la agricultura industrial globalizada tiene una creciente preeminencia.

Los métodos y los cálculos para la evaluación de externalidades sobre la agricultura industrial argentina permiten brindar inferencias sobre la precariedad de los métodos convencionales de cálculo agroproductivo en términos de las opciones productivas y su sustentabilidad. Pero no pueden incluir los costos generados por externalidades inciertas o irreversibles como la pérdida de biodiversidad o la desaparición del sustrato productivo tanto para la generación actual como para las generaciones futuras.

No obstante, se hace interesante contabilizar en términos físicos la movilidad de los distintos recursos involucrados en el suelo pampeano, no para su inclusión en los cálculos de costo-beneficio sino para su interpretación en términos del análisis de sustentabilidad, bajo un paraguas de relevamiento integrado de los recursos, como datos relevantes de indicadores biofísicos de (in)sustentabilidad y como un aporte también significativo hacia el decisor de turno de las políticas públicas.

El asumir la fuerte extracción determinada, en términos de los volúmenes exportados, a partir de mediados de los años noventa, generados por el cultivo de soja y 
la forma en que se está vaciando la caja de nutrientes de la región pampeana, puede entenderse como un aporte hacia los decisores de políticas y la sociedad argentina, que en muchos casos considera que estos recursos son ilimitados.

La valorización de los mismos en términos crematísticos (es decir, como lo diría la economía convencional) ha pretendido mostrar que si los costos de producción incluyeran las externalidades, éstas - en términos de fertilizantes a reponer, claramente mensurables - aportarían también a una organización más efectiva de los sistemas productivos y constituirían una nueva herramienta para comprender la sobreexplotación que enfrentamos.

Sin embargo, ha de verse que se debe insistir en los modelos de medición de extracción de nutrientes no incluyen en su evaluación de costos los importantes efectos que se producen por la alteración de los ciclos y las dinámicas particulares de cada nutriente. Los ciclos pueden sufrir modificaciones profundas en el tiempo y en el espacio y en distintos ambientes ecológicos.

La extracción de nutrientes puede comprenderse en términos de una aproximación a la evaluación de acercamiento o alejamiento a una sustentabilidad débil en la situación del suelo pampeano y como elemento importante a la definición de políticas ambientales sostenibles hacia el sector del que Argentina depende.

El análisis respecto a la salida y extracción de nutrientes no sólo intentó reflejar un nuevo y único indicador, sino manifestar y demostrar especialmente la preocupación sobre un modelo insostenible en el término de la utilización de los recursos del suelo, donde la soja, como monocultura, viene a representar un modelo de extracción degradatorio y de sobreexplotación pobremente analizado en toda su complejidad.

El caso de la cuenca del río Luján es útil para seguir investigando lo que sucede con una región que ha pasado rápidamente de una cultura de producción agropecuaria mixta a una intensificación tanto agrícola como ganadera en los usos del espacio productivo. El aumento de la carga de agroquímicos, el incremento de los niveles de contaminación, los efectos sobre la napa y sobre el recurso hídrico, en particular sobre áreas colindantes con poblaciones cercanas, harán que sea obligatorio seguir revisando los resultados de este modelo agroproductivo industrial intensivo que se expande en Argentina y que también amenaza la estabilidad socioambiental de otros países del Cono Sur de América. 
Andriulo, A. et al., Exportación y balance edáfico de nutrientes después de 80 años de agricultura continua, XIII Congreso Latinoamericano de la Ciencia del Suelo, Aguas de Lindota, Sao Paulo, 1996.

Barros, V., "Tendencias y cambio climático en la Argentina”, Reunión Anual de AAPRESID, Rosario, 2008: http://www.cima.fcen. uba.ar/ lcr/articulos/resumen.pdf.

Binimilis, R., Pengue, W. A. y Monterroso, I. "Transgenic treadmill", Responses to the emergence and spread of glyphosate-resistant Johnsongrass in Argentina, Geoforum (aceptado para publicación), 2009.

Buzzi, E. et al., La tierra. Para qué. Para quiénes. Para cuántos. Por una agricultura con agricultores, Buenos Aires, Ciccus, Fundación Centro Integral Comunicación, Cultura y Sociedad, 2005, p. 19.

Casas, R., "Causas y evidencias de la degradación de los suelos en la región pampeana", en Solbrig, O, Paarlberg, R y Di Castri, F (eds.), Globalization and the Rural Environment, The David Rockefeller Center Series on Latin American Studies, Cambridge, Harvard University Press, 2001.

Casas, R., Sustentabilidad de la agricultura en la regiónpPampeana, Buenos Aires, INTA Castelar, 2003.

Darwich, N., "Hasta cuándo puede expandirse el horizonte para la soja. Extracción y Balance de Nutrientes", boletín, Consejo Profesional de Ingeniería Agronómica, 2002.

Guichón, M. L., Angelini, B., Benítez, A., Serafini, M. y Cassini, M. H., "Caracterización ambiental de la cuenca del río Luján (Argentina) aplicando dos metodologías de procesamiento de información satelitaria", Revista de Teledetección, núm. 11, Buenos Aires, junio de 1999, pp. 1-7.

Flores, C. C. y Sarandón, S. J., “¿Racionalidad económica versus sustentabilidad ecológica? El ejemplo del costo oculto de fertilidad del suelo durante el proceso de agriculturización en la Región Pampeana, Argentina”, Revista de la Facultad de Agronomía, La Plata 105 (1), 2002.

INTA, "Sustentabilidad de la agricultura en la región pampeana", Buenos Aires, Instituto de Suelos, Castelar, mimeo, 2003.

Martínez Alier, J., De la economía ecológica al ecologismo popular, Montevideo, Ediciones Nordan Comunidad, Icaria, 1995, p. 143.

Curso de Economia Ecológica, Serie Textos Básicos para la Formación Ambiental, núm. 1., Programa de las Naciones Unidas para el Medio Ambiente. México, 1998, 164 pp.

"Ecología Industrial y Metabolismo Socioeconómico. Concepto y evolución histórica”, Economía Industrial, núm. 351, 2003.

Momo, F y otros, Estado ecológico de la cuenca del río Luján y utilidad de los indicadores biológicos para su control, mimeo, UNGS, s/f.

Morello, J. y Solbrig, O. (comps). ¿Argentina, granero del mundo: hasta cuándo?, Buenos Aires, Orientación Gráfica Editora, 1997, 280 pp.

Noticias Merceditas, "Relevamiento en establecimiento de feed lot en Jofré", Noticias Merceditas, 30 de enero de 2009.

Pagina 12, "La soja se quedó con las ganas", Buenos Aires, Pagina 12, 23 de diciembre de 2008.

Pengue, W. A., Cultivos transgénicos. ¿Hacia donde vamos?, Buenos Aires, UNESCO, 2000, 206 pp.

"Environmental and socioeconomic impacts of transgenic crops in Argentina and South America: An ecological economics approach", en Breckling, B. y Verhoeven, E. (eds.), Risk Hazard Damage. Specification of Criteria to Assess Environmental Impact of Genetically Modified Organisms, Bonn, Federal Agency for Nature Conservation, 2004

"Agricultura industrial y transnacionalización en América Latina. ¿La transgénesis de un continente?", Red de Formación Ambiental, Serie Textos Básicos para la Formación Ambiental, núm. 9, Programa de las Naciones Unidas para el Medio Ambiente, México, 2005, 220 pp.

"Transgenic crops in Argentina: the social and ecological debt", BSTS. Bulletin of Science, Technology \& Society, Canadá, agosto de 2005b.

"Modelo Agroexportador, Hidrovía Paraguay Paraná y sus consecuencias so- 
cioambientales. ¿Una compleja integración para la Argentina?", Una visión desde la economía ecológica y el enfoque multicriterial, Coalición Ríos Vivos, Rosario, Taller ecologista, mayo de 2006, 72 pp.

Sobreexplotación de recursos naturales y mercado agroexportador: hacia la determinación de la deuda ecológica con la Pampa Argentina, Córdoba, España, tesis doctoral, Junio 6. 2006 b, 303 pp.

"Agua virtual, agronegocio sojero y cuestiones económico ambientales futuras", Fronteras, 5:14-25, 2006c.

, La apropiación y el saqueo de la naturaleza. Conflictos ecológicos distributivos en la Argentina del Bicentenario, Buenos Aires, Lugar Editorial, 2008.

"La economía ecológica y el desarrollo en América Latina", Fronteras, 7 , GEPAMA. FADU. UBA, Buenos Aires. 2008: www.gepama.com.ar/fronteras.

"Agrofuels and Agrifoods.Counting the Externalities at the Major Crossroads of the 21st Century", Bulletin of Science,Technology \& Society, 2009.

Service, R.F., "A growing threat down on the farm", Science, núm. 316, pp. 1114-1117, 2007.
Toledo, V., "Metabolismos rurales: hacia una teoría económico-ecológica de la apropiación de la naturaleza", Revista Iberoamericana de Economía Ecológica, vol. 7, 1-26, Quito, 2008

Viglizzo, E. F y otros, La sustentabilidad ambiental del agro pampeano, Buenos Aires, Ediciones INTA, 2002.

Vulcano, P., "La contaminación llegó al campo", Revista Noticias, Buenos Aires, 2006.

Acrónimos

AAPRESID. Asociación Argentina de Productores en Siembra Directa.

CREA. Consorcio Regional de Experimentación Agrícola.

FAA. Federación Agraria Argentina.

ICO. Instituto del Conurbano. Universidad Nacional de General Sarmiento.

INPOFOS. Instituto de la Potasa y el Fósforo.

INTA. Instituto Nacional de Tecnología Agropecuaria.

SAGPYA. Secretaria de Agricultura, Ganadería, Pesca y Alimentación. 